Post-print copy of [B Samel, N Sandström, P Griss, and G Stemme, Fellow, IEEE, " Liquid Aspiration and Dispensing Based on an Expanding PDMS Composite", J. Microelectromechanical systems, 17, 5, 2008]

doi:10.1109/JMEMS.2008.921728

website: http://ieeexplore.ieee.org/xpl/articleDetails.jsp?arnumber=4580118 


\title{
Liquid Aspiration and Dispensing Based on an Expanding PDMS Composite
}

\author{
Björn Samel, Niklas Sandström, Patrick Griss, and Göran Stemme, Fellow, IEEE
}

\begin{abstract}
In this paper, we present the development of active liquid aspiration and dispensing units designed for vertical, as well as lateral, liquid aspiration. The devices are based on a single-use thermally expanding polydimethylsiloxane (PDMS) composite, which allows altering its surface topography by means of individually addressable integrated heaters. Devices are designed in order to create an enclosed cavity in the system, due to locally expanding the initially unstructured composite. This enables negative volume displacement and leads to the event of liquid aspiration. To enable this device functionality, two different techniques of selectively creating permanent PDMS bonds have been developed. One approach utilizes the plasma-assisted PDMS bonding technique, together with a patterned antistiction layer to form reversibly, as well as irreversibly, bonded regions. Another approach utilizes microcontact printing of PDMS curing agent, which serves as a patterned intermediate layer for adhesive bonding. Fabricated prototype devices successfully demonstrated the aspiration and release of liquid volumes ranging from 28 to $815 \mathrm{~nL}$. The devices are entirely fabricated from low-cost materials, using wafer-level processes only and do not require external means for liquid actuation.
\end{abstract}

manner. In early developments, materials well known from the conventional semiconductor industry, such as silicon and glass, were used [4]-[6]. However, the complexity of devices based on these materials and their associated fabrication techniques make integration into such systems difficult and costly. Ideally, such components are easy to integrate, straightforward to fabricate, easy to use, robust, free of dead volume, and cheap to duplicate.

Therefore, polymer microfabricated devices based on new materials and actuation methods have gained increased attention from the MEMS society. The use of polymers is very attractive for a number of reasons. They are cheap compared to single-crystalline silicon and easy to process allowing various molding and embossing techniques to be used. Moreover, they offer the possibility to manipulate physical and chemical properties.

Several promising polymer-based actuation principles have recently been presented, addressing the aforementioned issues, which potentially promote the development of integrated microfluidic systems. They exploit a variety of both mechanical and chemical actuation principles, such as external pressure sources [7], [8], stimuli responsive polymers [9]-[11], centrifugal forces on a rotating disk [12], electrochemical gas generation through electrolysis [13], [14], decomposition of hydrogene peroxide [15] or azobis isobutyronitrile [16], melting of paraffin [17], thermal expansion of gas [18], [19], or expansion of a condensed gas encapsulated in chemically inert microspheres [20].

Until now, the issue of introducing a liquid sample into a microfluidic chip in a convenient manner has often been neglected. The proposed actuation principles mostly focus on the dispensing of liquid volumes and not on their aspiration. This complicates the development of microfluidic systems that are capable of conducting the standard analytical procedures in an autonomous, accurate, reliable, and convenient manner. Therefore, the issue of introducing a liquid sample into a device and its subsequent transport, e.g., into a reaction compartment, must be addressed for the development of technically mature systems. Most presented approaches for liquid aspiration are based on the traditional MEMS materials and actuation principles, e.g., piezoactuators [21]-[23], suffering from the before mentioned disadvantages. Moreover, they require the use of external equipment, such as stepper motors, controllers, and vacuum or syringe pumps.

Our group recently presented a thermally responsive polydimethylsiloxane (PDMS) composite and the development of single-use liquid handling components thereof. The composite consists of expandable microspheres incorporated into a PDMS matrix and facilitates the development of microfluidic valves pumps, valves, and mixers, are integrated on a single crofluidic chip in order to manipulate liquids in a controlled 
[24], pumps [25], and fully integrated disposable biochips [26]. Due to the hydrophobic nature of PDMS-based microfluidic devices, the introduction of a liquid sample cannot be carried out using capillary filling. Instead of that, external vacuum pumps are often used for sample loading, which is time consuming and inapplicable for point-of-care applications.

In this paper, however, we demonstrate for the first time active liquid aspiration and release thereafter by means of locally altering the surface topography of an initially unstructured thermally expanding PDMS composite. This is accomplished via two novel PDMS bonding techniques which enable the formation of a permanent bond at designated regions on the PDMS chip. The applicability of the selective PDMS bonding techniques is shown by means of fabricating devices for controlled in-plane and out-of-plane liquid volume aspiration and release. The electrically controllable devices are easy to integrate into microfluidic networks, do not require external actuators for liquid control and are based on low-cost materials and waferlevel processes only. These devices are intended for use in applications in the medical or biological field. Although previous work has been presented, where biosamples have been manipulated effectively, it is not proven that the devices work reliably when manipulating samples containing protein or DNA molecules. Yet, the liquid handling concept potentially enables the development of autonomous systems for controlled and costeffective transdermal microfluidic applications, point-of-care diagnostics, disposable biochips, or lab-on-a-chip applications.

\section{DESIGN IsSUES}

The proposed novel liquid handling concept for liquid aspiration is based on a PDMS composite which expands upon heating and, thus, allows locally tailoring its surface topography to achieve a desired profile without previously patterning it. Liquid aspiration and release are achieved in vertical, as well as lateral, direction by patterning the composite accordingly to form an enclosed cavity in the system, i.e., inverse volume displacement. Precise spatial and temporal control of the composite expansion are achieved by means of lithographically defined integrated heaters. During device operation, the cover lid is being lifted upwards upon the event of aspiration and eventually a cavity is being created [Fig. 1(a)/(b)]. To enable this lifting procedure and accomplish cavity formation for both in-plane and out-of-plane liquid handling, several fabrication and design aspects have to be addressed.

First, an appropriate cover lid needs to be implemented. A lid made of only PDMS would be too flexible, due to its elastomeric nature, and would not allow adequate lifting during operation to form a cavity. Using glass as the cover material would restrict the wafer-level fabrication process of the devices. Instead, a reinforced cover lid is fabricated by attaching a 3M-polyester-film to a PDMS layer. This counteracts the low Young's modulus of PDMS giving additional stiffness to the cover lid, which enables its lifting during operation and retains its straightforward fabrication characteristics.

Second, to ensure device functionality, the reinforced cover lid and the bottom part of the device have to be bonded together in such a manner that there are reversibly, as well as irreversibly, (a)

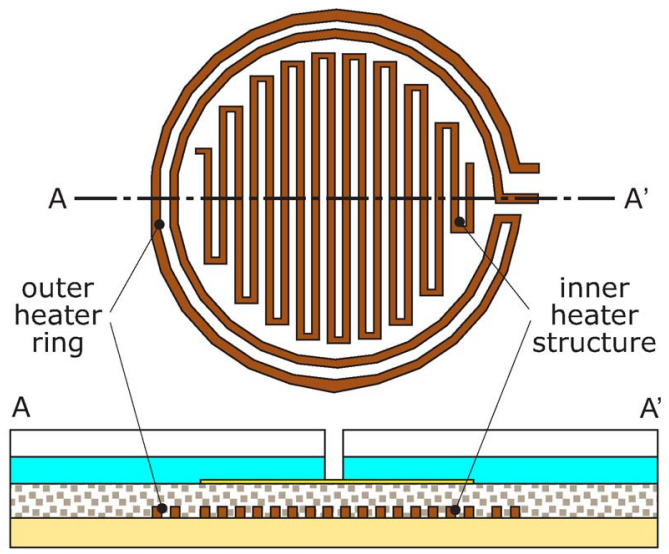

(b)

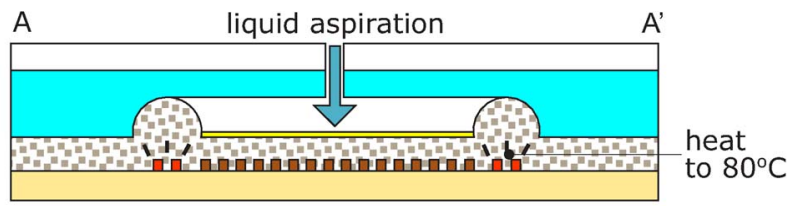

(c)

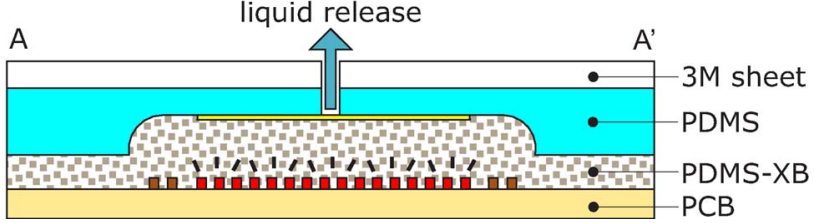

Fig. 1. (a) Schematic of the working principle showing the layout of the heater structures as well as the cross-sectional view along the cutting line AA' of a single device. (b) Upon triggering the outer heater ring the PDMS-XB composite expands, and as a consequence, a cavity is being created which leads to liquid aspiration through the hole in the lid. (c) Subsequently, triggering the inner heater structure leads to the release of the afore aspirated liquid.

bonded areas on the same wafer. To achieve an area where no bonding occurs, i.e., the area where a cavity is being created during device operation, formation of a permanent bond at these specific positions has to be prevented in order to avoid impairing the lifting of the cover lid. To be able to bond PDMS layers selectively to one another, we developed two different bonding processes which allow to bond PDMS structures selectively on full-wafer scale. One technique utilizes the widely used PDMS plasma bonding process, where a patterned antistiction layer is introduced to the PDMS surfaces prior to plasma exposure. This prevents areas from being oxidized during the plasma oxidation step. Here, we use $\mathrm{Au}$ as the material for the antistiction layer. Another approach utilizes a novel full-wafer adhesive bonding technique where PDMS curing agent is used as the intermediate layer for adhesive bonding. This technique has shown to create bonds as strong as, or even stronger than, the widely used plasma-assisted PDMS bonding technique [27]. To selectively bond PDMS by means of this technique, we utilize here a softlithographic approach of microcontact printing PDMS curing agent onto one of the PMDS surfaces. The transferred curing agent represents a patterned intermediate layer for adhesive bonding, which fulfills the bonding function at specific regions after curing.

To show the applicability of both bonding techniques, the $\mathrm{Au}$ antistiction layer is used for fabrication of out-of-plane devices, whereas the technique of microcontact printing of PDMS curing agent is used for fabrication of in-plane liquid handling devices. The response of the devices is highly dependent on the 
(a)

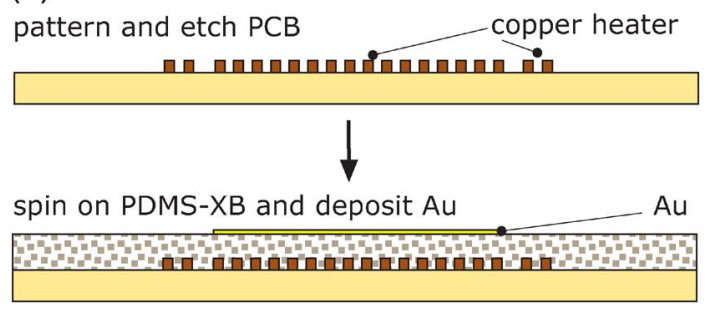

(b)

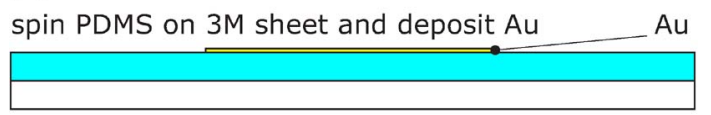

(c)

plasma bonding

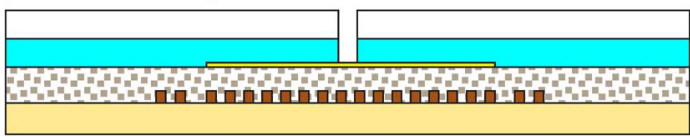

Fig. 2. Process flow of the principal fabrication steps: (a) First, a PCB is lithographically patterned and the PDMS-XB composite subsequently spun on and cured. (b) Reinforced cover lid is fabricated by spinning PDMS on a $3 \mathrm{M}$-sheet. Additionally, Au patterns are defined on both the cover lid and the stack of PCB/PDMS-XB. (c) Cover lid from (b) is then plasma bonded to the fabricated stack from (a), and access holes are punched out of the cover lid.

power input, composite thickness, amount of expandable microspheres in the composite layer, substrate material, and heater geometry. The size of the resulting cavity, and in turn the liquid volume which eventually is being aspirated, is determined by the dimensions of the unbonded area, the mixing ratio of the composite actuator, as well as the size of the integrated heaters.

\section{VERTICAL ASPIRATION}

\section{A. Principle of Operation and Design}

Integrated heaters are designed to consist of an outer heater ring and an inner heater structure [Fig. 1(a)]. The principle of operation for vertical aspiration and release is shown in Fig. 1 for two different actuation steps. The main characteristic of the composite actuator is to expand upon heat, and when adequate voltage is applied to the outer heater ring, the composite expands along this heater geometry, which results in lifting of the reinforced cover lid and, hence, formation of a cavity [Fig. 1(b)]. As a consequence of this inverse volume displacement, the cavity fills with liquid which is present at the access port. Addressing the inner heater of the device subsequently results in composite expansion into the cavity and release of the previously aspirated liquid volume, as shown in Fig. 1(c).

\section{B. Fabrication}

The devices are straightforward to fabricate and entirely made of low-cost materials. All photolithographical fabrication steps use high-resolution transparencies as photomasks. The principal fabrication steps are shown in Fig. 2. First, microheater patterns, featuring a ring heater and an inner heater structure, are lithographically defined on standard FR4 printed circuit boards (PCBs) to achieve localized heating of the composite actuator [Fig. 2(a)]. The composite is obtained by thor-

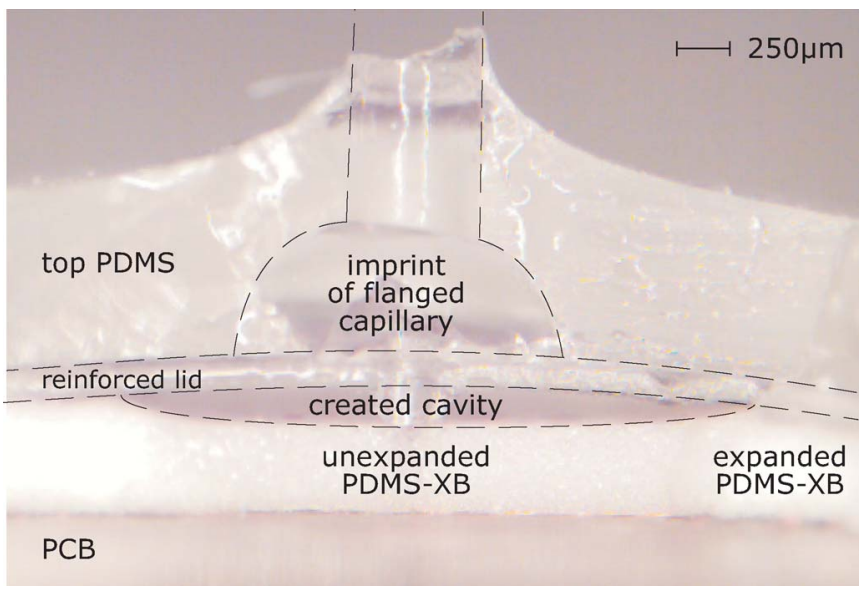

Fig. 3. Photograph showing the cross section of a single device after aspiration. Clearly visible is the created cavity which would lead to liquid aspiration through the flanged capillary tubing.

oughly mixing PDMS base, curing agent (10:1), and expandable microspheres $(\mathrm{XB})$ at a ratio of $300 \mathrm{mg} / \mathrm{mL}$ liquid PDMS prepolymer. A layer of the PDMS-XB composite is then spun on the patterned PCB at $400 \mathrm{r} / \mathrm{min}$ and cured at $65^{\circ} \mathrm{C}$. PDMS is supplied by Dow Corning (Sylgard 184 Silicone Elastomer Kit) and expandable microspheres by Expancel (Expancel 820DU).

During device operation, the cover lid is being lifted upwards upon the event of aspiration, and eventually, a cavity is created. To enable this lifting procedure, an appropriate cover lid is implemented by spinning liquid PDMS prepolymer on a sheet of Scotchpak 1022-release-liner $3.0 \mathrm{mil}(3 \mathrm{M})$ at $2000 \mathrm{r} / \mathrm{min}$, counteracting the low Young's modulus of PDMS and reinforcing the cover lid [Fig. 2(b)].

Second, an antistiction pattern is introduced by sputtering $\mathrm{Au}$ in the nanometer range through a mechanical mask on both the cover lid and the stack of PCB/PDMS-XB [Fig. 2(a)/(b)]. The Au-pattern prevents the formation of a permanent bond at these designated positions by preventing the surface to be oxidized properly during the subsequent plasma oxidation step.

Finally, access holes are punched through the cover lid, which is then bonded to the previously fabricated stack of PCB/PDMS-XB by oxidizing both layers in oxygen plasma, aligning and bringing them into conformal contact [Fig. 2(c)]. At the positions where $\mathrm{Au}$ is present, no permanent bond is formed. This eventually allows the detachment of the cover lid from the PCB/PDMS-XB stack and the formation of a cavity during operation.

\section{Experimental}

For liquid displacement evaluation purposes, fluidic interconnects are integrated by locating flanged capillary tubing on top of the access holes. Syringe needles are inserted through these interconnects and the previously punched out access holes. The inserted needles form a mechanical seal toward the access holes and at the same time provide foothold for the interconnects when casting additional PDMS over the structures. Removal of the needles enables filling of the aspiration ports under vacuum with glycol- and ethanol-based colored dye (red) diluted in deionized water. Fig. 3 shows a cross-sectional view 

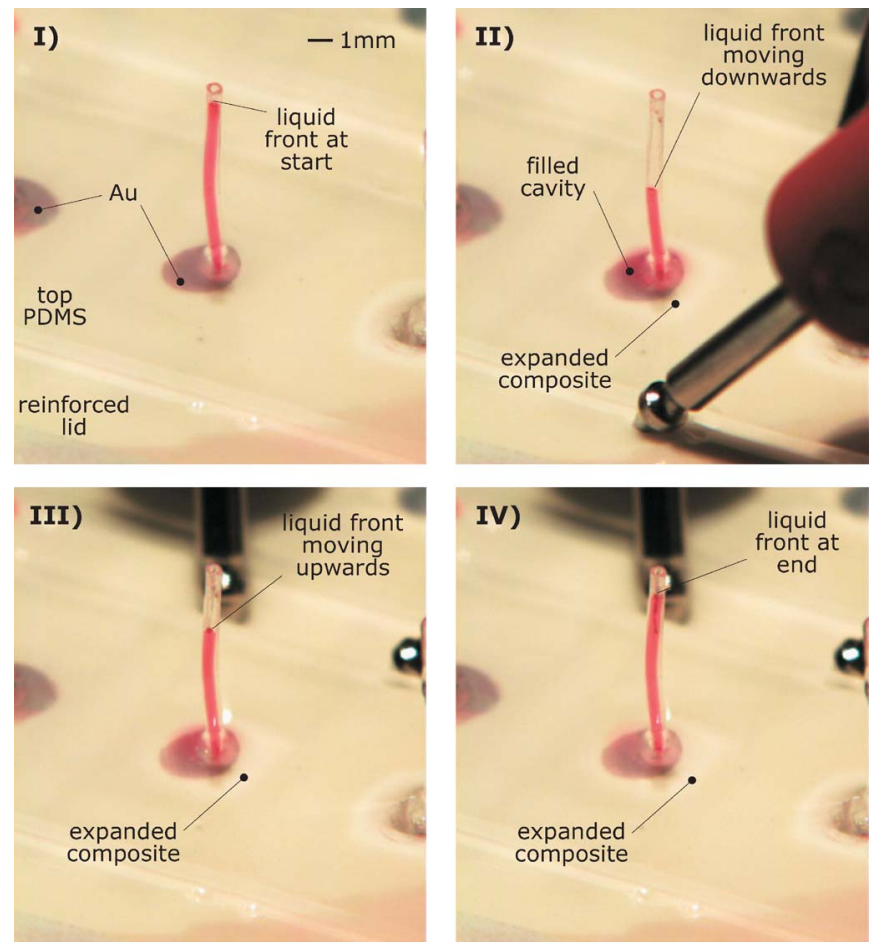

Fig. 4. Picture sequence showing the operation scheme of a device on a wafer: I) filled capillary tubing after sample loading; II) triggering the outer heater ring leads to liquid aspiration into the created cavity with liquid front moving downwards; III) subsequent liquid release by triggering the inner heater and liquid front moving upwards; IV) liquid front is almost back to its original position.

of the device after the event of aspiration and removal of the fluidic interconnect.

The picture sequence [Fig. 4(I)-(IV)] shows liquid aspiration and subsequent release from the device. Applying adequate voltage to the outer heater ring results in liquid aspiration [Fig. 4(II)]. The liquid front starts to move downwards and the cavity fills with liquid. Subsequently, triggering the inner heater results in liquid release from the cavity, as a consequence which is depicted in Figs. 4(III) and (IV), where the liquid front has moved upwards. The heaters were driven with a mean power of $800 \mathrm{~mW}$ for 10 and $5 \mathrm{~s}$, respectively.

Capillary tubing was calibrated by means of a syringe pump prior to measurements and connected to the devices to conduct volume measurements. The advancing liquid meniscus inside the capillary tubing was used as the measure of displaced liquid. The displacement characteristics were evaluated by recording the experiment using a microscope and charge-coupled device (CCD) camera and, thereafter, conducting image analysis using Matlab software. Fig. 5 shows results of consecutively measured liquid volumes that were aspirated and subsequently released from prototypes with integrated heaters of two different sizes. A first set of devices, with an outer heater ring diameter of $4.5 \mathrm{~mm}$, showed aspiration of a mean liquid volume of $815 \mathrm{~nL}$, with a relative standard deviation of $4.9 \%$. Subsequent liquid release showed a mean liquid volume of $747 \mathrm{~nL}$, with a relative standard deviation of $8.7 \%$. Another set of devices, with an outer heater ring diameter of $3 \mathrm{~mm}$, aspirated a mean liquid volume of $180 \mathrm{~nL}$, with a relative standard deviation of $16.6 \%$ and subsequently released mean liquid volume of $162 \mathrm{~nL}$, with

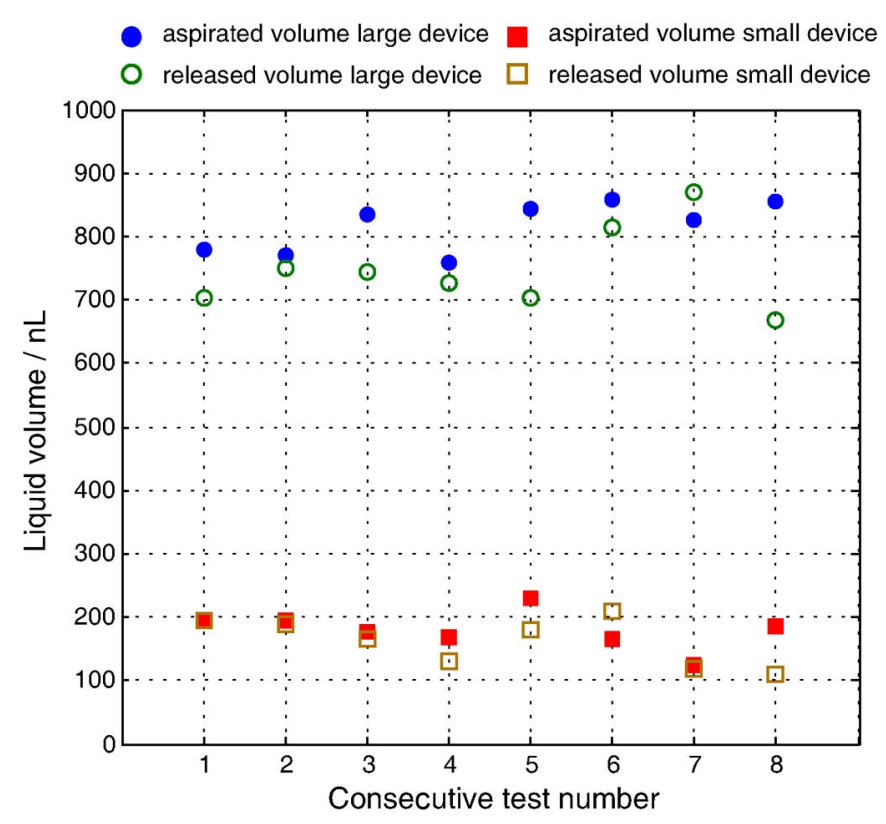

Fig. 5. Graph showing the result of aspirated liquid volumes with subsequent liquid release for a set of differently sized devices which have been used consecutively.

a relative standard deviation of $23.6 \%$. The dead liquid volume as well as the relatively high standard deviation, particularly for the smaller devices, might be related to the prototype nature of the devices. Possible sources that contribute to this error could be related to fabrication issues, such as quick alignment of the layers after plasma oxidation, use of nonoptimized inner heater structures or the manual assembly of fluidic interconnects.

\section{LATERAL ASPIRATION}

\section{A. Principle of Operation and Design}

Similarly to the previous design, a cover lid is being lifted upwards upon the event of aspiration, and consequently, a cavity is created [Fig. 6(a)/(b)]. The approach of reinforcing the cover lid is applied in this design as well, but additionally contains an imprinted microchannel. The path of this integrated microchannel is interrupted at the location where the cavity is created during operation, constituting a barrier in the microchannel. This barrier is reversibly bonded to the bottom structure to enable out-of-plane movement. To form such a reversibly bonded barrier an adhesive PDMS bonding technique [27] is applied, which utilizes a soft-lithographic approach of microcontact printing PDMS curing agent to accurately achieve a patterned intermediate layer for adhesive bonding, defining bonded and unbonded areas in the system.

For active in-plane liquid aspiration through the microchannel inlet and subsequent liquid release through the microchannel outlet, integrated valves have to be adapted in order to change the state of the channel inlet and outlet from open to closed and vice versa. The heater design consists of integrated inlet and outlet valve heaters, outer heaters as well as an inner heater structure. The initial state of the device at the channel inlet is normally open, whereas the channel outlet is normally closed, prior to liquid aspiration. In case the channel 


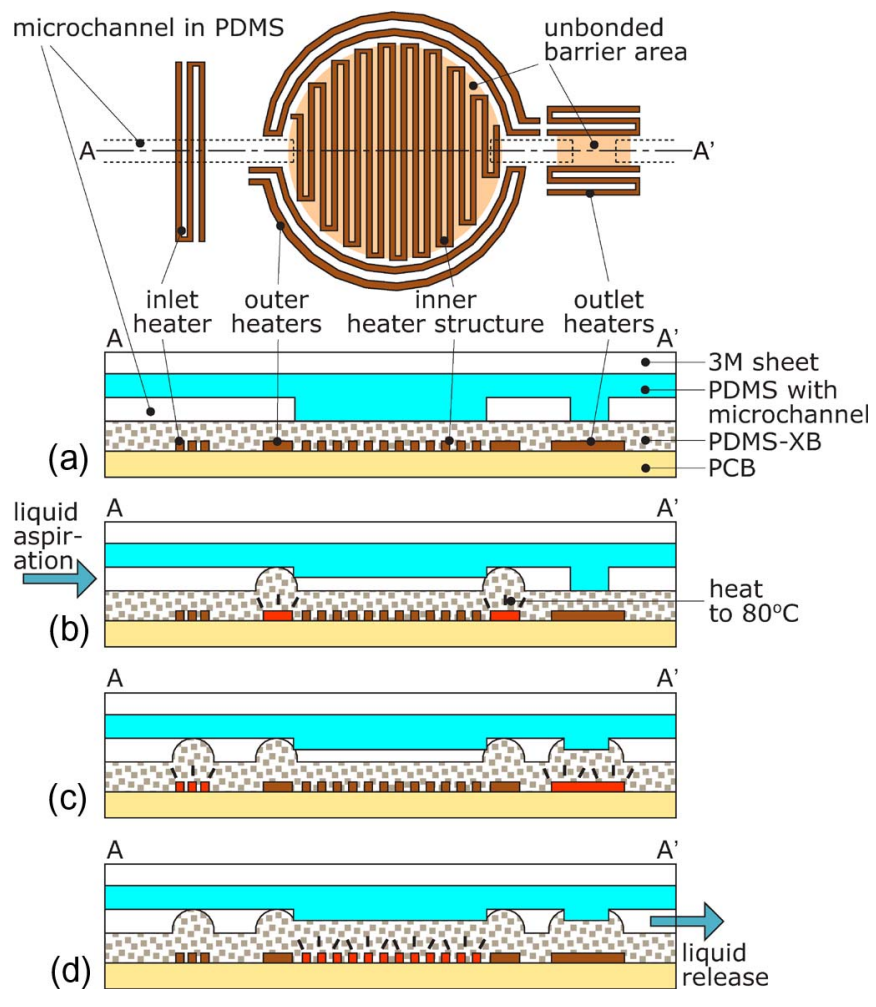

Fig. 6. (a) Schematic of the working principle showing the layout of the heater structures and microchannel with its barriers as well as the cross-sectional view along the cutting line AA'. (b) Upon triggering the outer heaters the PDMS-XB composite expands, and as a consequence, a cavity is being created which leads to liquid aspiration through the inlet. (c) Subsequently, triggering the valve heaters leads to closing and opening of inlet and outlet channel, respectively. (d) Afore aspirated liquid is released into the outlet channel upon triggering the inner heater.

outlet is open upon the event of liquid aspiration, the inverse volume displacement would be insufficient and, in turn, lead to incomplete filling of the cavity. This normally closed state of the channel outlet can be realized in two different ways.

One approach utilizes a supplementary barrier in the microchannel outlet, as shown in Fig. 6. At this position, the interface to the PCB/PDMS-XB stack is bonded reversibly similar to the area where the cavity is created during operation. Such a design constitutes a normally closed valve that utilizes two microheaters positioned nearby the supplementary barrier along the microchannel acting as outlet heaters [Fig. 6(a)]. To induce liquid aspiration, adequate voltage is applied to the outer heaters, which makes the composite expand along this heater geometry resulting in lifting of the reinforced cover lid. As a consequence, this cavity fills with liquid which is present at the microchannel inlet [Fig. 6(b)]. Subsequently, addressing the inlet valve heater results in closing off the microchannel inlet [Fig. 6(c)]. To remove the supplementary barrier at the channel outlet, the two outlet heaters along the microchannel are triggered, which in turn leads to opening of the channel outlet by lifting the supplementary barrier [Fig. 6(c)]. Triggering the inner heater of the device results in composite expansion into the cavity and release of the previously aspirated liquid volume through the channel outlet, as shown in Fig. 6(d).

Unlike the previous outlet valve, where the outlet heaters were placed along the microchannel, another approach utilizes

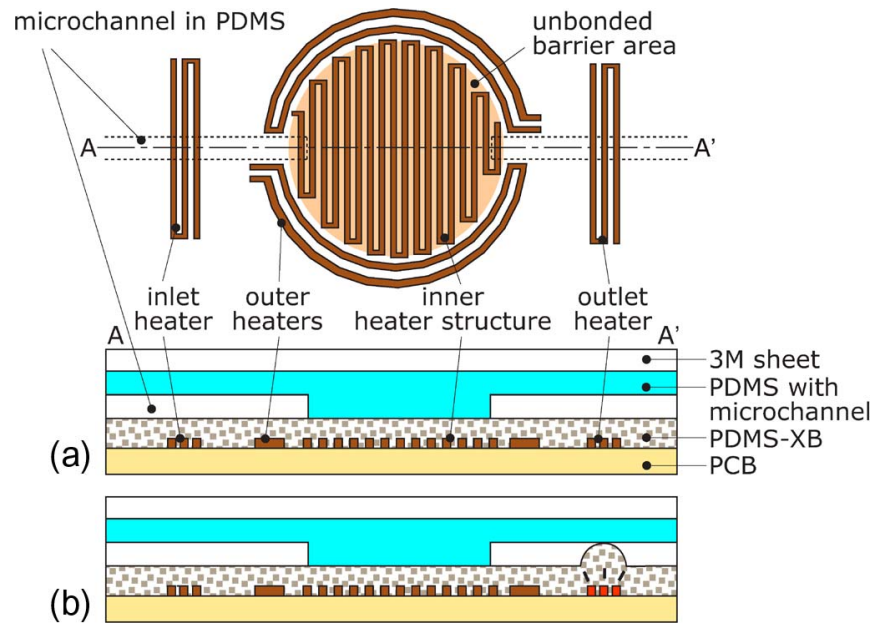

Fig. 7. (a) Schematic of the design where a miroheater is placed perpendicularly to the microchannel outlet. (b) Triggering this heater closes the microchannel outlet, and the device can be used for liquid aspiration. The subsequent operation steps are similar to the previous design.

a microheater placed perpendicularly across the microchannel outlet, which is similar to the inlet valve heater, as shown in Fig. 7. Triggering this heater closes the outlet channel. As reported in [24], closed microchannels using such valves can be reopened by applying pressure on one side. This breakthrough pressure is highly dependent on the total energy input. In this design, with two similar valves at the channel inlet and outlet, liquid flow can be directed by diversifying the energy contribution between the valve heaters. When triggering the inner heater structure subsequently, the generated pressure will reopen the one valve with the lower energy contribution, and liquid is released into this microchannel. To support the directional liquid transportation into a microchannel, the inner heater is designed as a decreasingly dense heater meander [26].

\section{B. Fabrication}

The straightforward fabrication of devices is retained in this design, which involves low-cost materials only and highresolution transparencies as photomasks. Fig. 8 shows the principal fabrication steps. Microheater patterns featuring outer heaters, inner heater, and valve heater structures are lithographically defined on standard FR4 PCBs to achieve localized heating of the composite actuator [Fig. 8(a)]. The composite is obtained by thoroughly mixing PDMS base, curing agent (10:1), and expandable microspheres at a ratio of $200 \mathrm{mg} / \mathrm{mL}$ liquid PDMS prepolymer. A layer of the PDMS-XB composite is then spun on the patterned PCB at $400 \mathrm{r} / \mathrm{min}$ and cured at $65{ }^{\circ} \mathrm{C}$ for $4 \mathrm{~h}$.

Next, a reinforced cover lid with integrated microchannels imprinted in PDMS is fabricated through replica molding. A master is fabricated by spinning Shipley SJR 5740 photoresist on a silicon wafer at $800 \mathrm{r} / \mathrm{min}$, to yield $20 \mu \mathrm{m}$ high structures. Subsequent heating to $200{ }^{\circ} \mathrm{C}$ makes the photoresist reflow, which results in structures that are curved at the edges. The curved edges ensure complete valve sealing during operation of the device. Liquid PDMS prepolymer is spun onto the master at $750 \mathrm{r} / \mathrm{min}$, a sheet of Scotchpak-9742-release-liner $4.6 \mathrm{mil}$ 
(a)

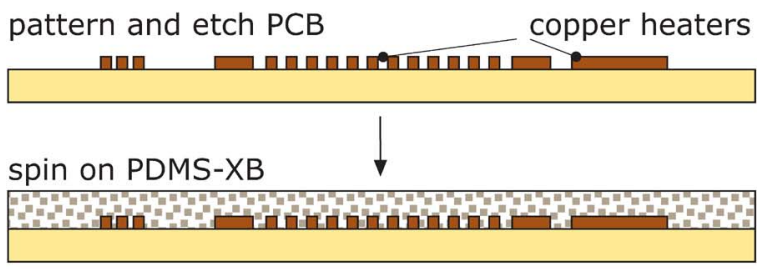

(b)

spin PDMS on resist master and apply 3M sheet

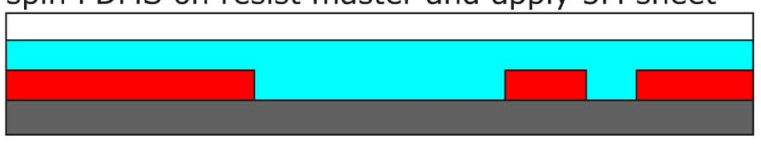

(c)

fabricate PDMS stamp on SU-8 master

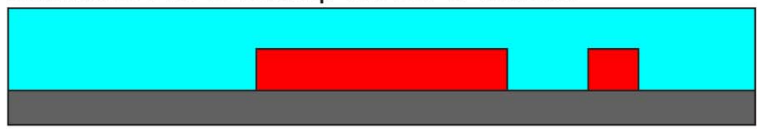

(d)

transfer curing agent to stamp

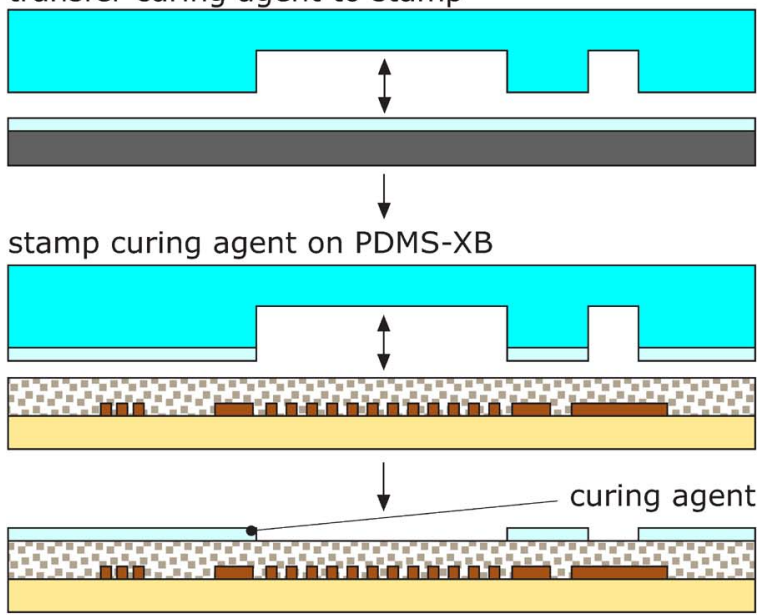

(e)

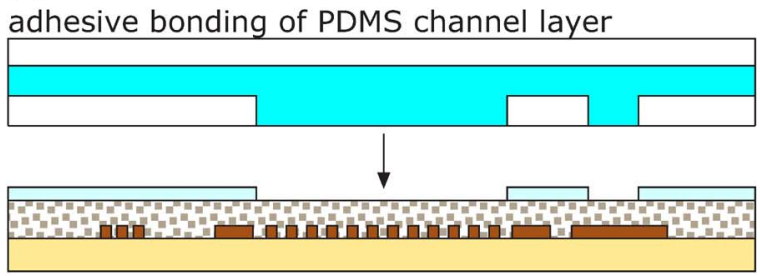

(f)

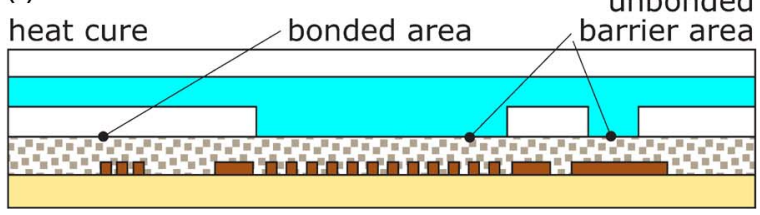

Fig. 8. Process flow of the principal fabrication steps: (a) First, a PCB is lithographically patterned and the PDMS-XB composite subsequently spun on and cured. (b) Reinforced cover lid with integrated microchannels is fabricated by spinning PDMS on a resist mold and adding a 3M-sheet. (c) PDMS stamp is fabricated by casting PDMS over an SU-8 mold. (d) PDMS curing agent is spun on a substrate and transferred to the previously fabricated stack of PCB/ PDMS-XB. (e) Cover lid with integrated microchannels from (b) is then aligned and placed onto the PCB/PDMS-XB/curing agent stack from (d). (f) Finally, the device is heat cured to generate a permanent bond between the layers.
(3M) is placed on top, followed by a degassing step in vacuum and heat curing [Fig. 8(b)]. Adding the 3M sheet counteracts the low Young's modulus of PDMS, giving additional stiffness to the cover lid. This enables its lifting during operation [Fig. 6(b)], since a lid made of only PDMS would be too flexible and thus impede the lifting process during operation.

To bond together the cover lid and the previously fabricated stack of PCB/PDMS-XB, we utilize a selective PDMS adhesive bonding technique via microcontact printing of PDMS curing agent. To fabricate a PDMS stamp for microcontact printing, we use replica molding. A master is fabricated by spinning SU-8 2100 (Microchem) at $2000 \mathrm{r} / \mathrm{min}$, and stamp regions are defined photolithographically. Liquid PDMS prepolymer is cast over the master, cured at $110^{\circ} \mathrm{C}$ for $20 \mathrm{~min}$ and eventually peeled off [Fig. 8(c)]. PDMS curing agent, used as the intermediate layer for adhesive bonding, is spun on a substrate at $6000 \mathrm{r} / \mathrm{min}$, as shown in Fig. 8(d). Then, the fabricated PDMS stamp is pressed on the coated substrate, to pick up the curing agent, and placed on the previously fabricated stack of PCB/PDMS-XB. This renders areas of the PCB/PDMS-XB stack coated with curing agent, according to the "inked" stamp pattern. Prototypes of different sizes are fabricated by changing the dimensions of the PDMS stamps which are used for microcontact printing of PDMS curing agent as well as adapting the dimensions of the respective heaters. Finally, the cover lid is aligned and placed on the stack of PCB/PDMS-XB, as shown in Fig. 8(e). A permanent bond is accomplished at the interface where curing agent is present by heat curing at $65^{\circ} \mathrm{C}$ for $4 \mathrm{~h}$ [Fig. 8(f)].

\section{Experimental}

For liquid displacement evaluation purposes, the inlet channel is filled under vacuum with glycol- and ethanol-based colored dye (red) diluted in deionized water. Fig. 9(I) shows the initial structure after filling prior to device operation. The picture sequence [Fig. 9(I)-(IV)] shows active in-plane liquid aspiration and subsequent release from the device. Applying adequate voltage to the outer heaters results in liquid aspiration through the channel inlet. Fig. 9(II) shows the generation of a cavity being filled with liquid which is present at the channel inlet. Subsequently, triggering the valve heaters leads to closing off and opening the inlet and outlet channel, respectively [Fig. 9(III)]. This enables liquid release of the earlier aspirated liquid volume through the channel outlet by triggering the inner heater of the device, as shown in Fig. 9(IV). The heaters were driven at an average power of $700 \mathrm{~mW}$ for 8 and $4 \mathrm{~s}$, respectively.

Volume measurements were conducted, using the advancing liquid meniscus inside the microchannel as the measure of displaced liquid. The displacement characteristics were evaluated by recording the experiment, using a microscope and CCD camera and thereafter conducting image analysis using Matlab software. Experiments on liquid aspiration and subsequent release were conducted on at least three devices of each size. Fig. 10 depicts the released liquid volumes, ranging from 28 to $476 \mathrm{~nL}$, as a function of different stamp cavity diameters used for the fabrication of the prototypes. The results indicate improved liquid handling accuracy compared to the vertically 

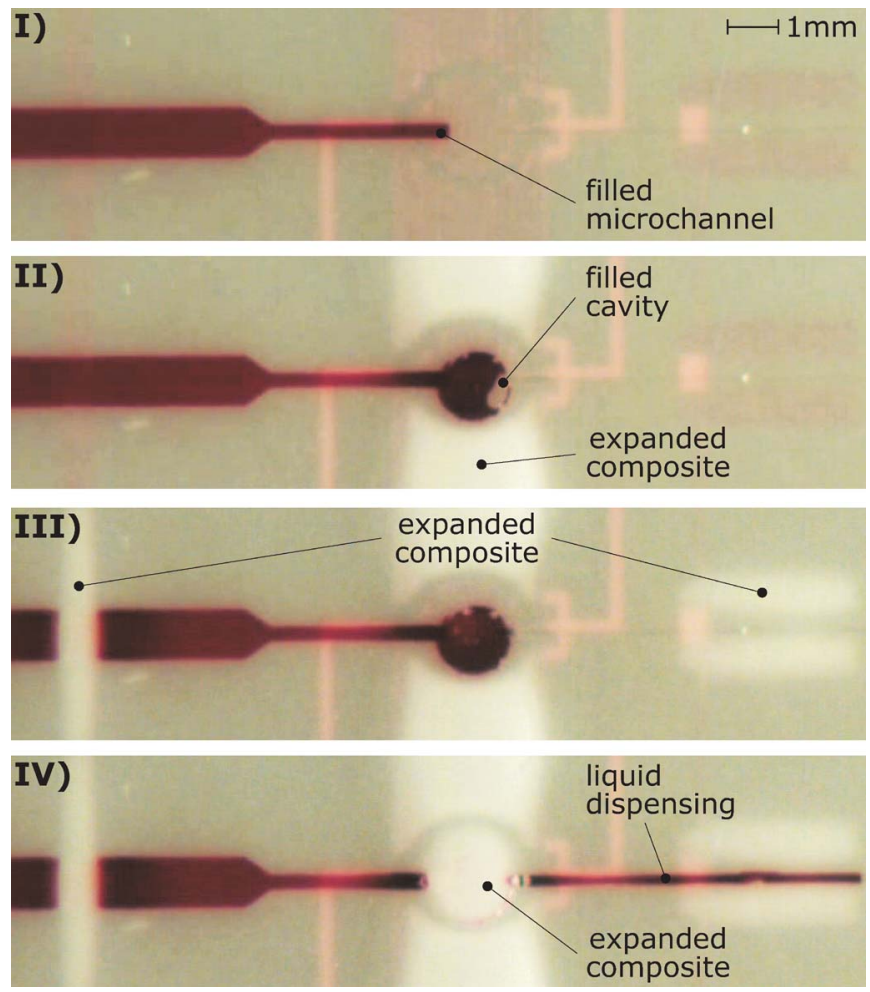

Fig. 9. Picture sequence showing the device under operation: I) filled microchannel inlet after sample loading in vacuum; II) triggering the outer heaters leads to liquid aspiration into the created cavity; III) subsequently, triggering all valve heaters closes of the channel inlet and opens the channel outlet; IV) triggering the inner heater results in dispensing of the afore aspirated liquid volume into the microchannel outlet.

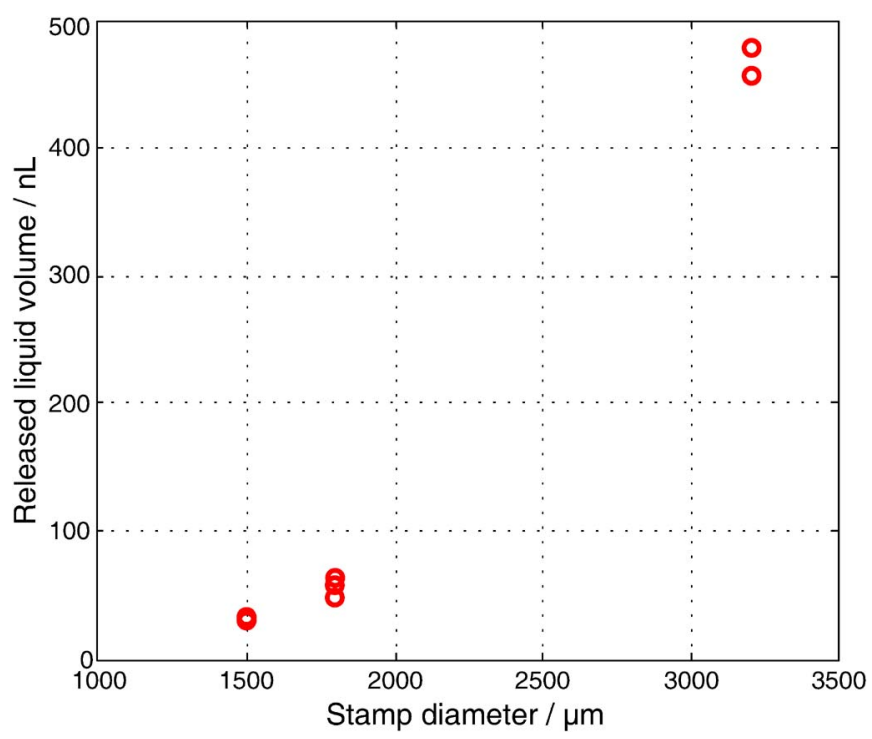

Fig. 10. Graph showing the released liquid volume after liquid aspiration for differently sized devices. The stamp diameters used for microcontact printing of curing agent accurately determines the dimension of the resulting cavity.

operating units. This can be related to higher accuracy during the evaluation experiment as well as the adhesive bonding approach, which offers improved layer alignment during fabrication, influencing the final device performance. No values were obtained for liquid volume aspiration, since the experimental setup did not allow easy quantification.

\section{Discussion AND OUtLook}

Several possibilities are described in order to optimize device performance concerning power consumption, liquid handling accuracy, and thermal load on the manipulated liquid volume. Several design parameters are of interest when configuring aspiration units based on the composite actuator, such as heater design, substrate material, initial composite thickness, and the ratio of expandable microspheres. Particularly interesting, from a device performance point of view, are the design parameters concerning the integrated heaters. Performance issues of the devices can further be related to the selection of appropriate fabrication techniques and materials. The presented prototypes are based entirely on low-cost materials and processes, which has implications on the practically achievable resolution during fabrication. The layer thickness of standard PCBs and the limited resolution of the integrated heaters lead to rather highenergy consumption during operation. A straightforward way of improving the energy consumption could be by choosing another substrate material, such as COC or COP, with a thin metal heater patterned on top, at high resolution. Such highresolution heater structures would also increase the precision of the composite expansion.

When evaluating the techniques of bonding PDMS selectively to another, the use of microcontact printing PDMS curing agent is advantageous compared to the plasma-assisted bonding technique. Using adhesive bonding with PDMS curing agent allows proper alignment of the structures, since bond formation is initiated upon heat. When using plasma bonding instead, technical skill is required to align two surfaces and bring them into contact after oxidation, since the oxidized PDMS surface reconfigures quickly in air. Furthermore, this approach potentially allows the functionalized immobilization of biomolecules inside the devices and prevents cross-contamination prior to device usage, since the fluidic pathway can be generated on demand. This is attractive for applications in the medical field.

From an environmental stability point of view, the presented devices can be compared to other PDMS-based applications and studies recently presented in literature [7], [8], [28].

The thermal load on the liquid is an issue that needs to be addressed when using active biosamples. Increased temperature could possibly lead to, e.g., cell lysis, protein degradation, or nucleic acid denaturation. The temperature range, in which a sample can be manipulated without negative effects, must be considered for each specific application. From that, a selection of design parameters and microfluidic components can be made for a potential biochip. The presented liquid aspiration concept offers to manipulate samples that allow temperature fluctuations. However, if a specific application requires a stable temperature of, e.g., $37^{\circ} \mathrm{C}$, the use of on-chip buffer liquids is suggested and/or the devices need to be optimized accordingly. [25], [26]. The heater structures in the present design are situated adjacent to the fluidic pathway rather than directly beneath it. A potential design, where a microchannel can be created on demand, is shown in Fig. 11, and consists of an array of heaters. By triggering the first set of heaters prior to the application of liquid at the inlet port, an airplug can be aspirated for increased thermal isolation, followed by the aspiration of 


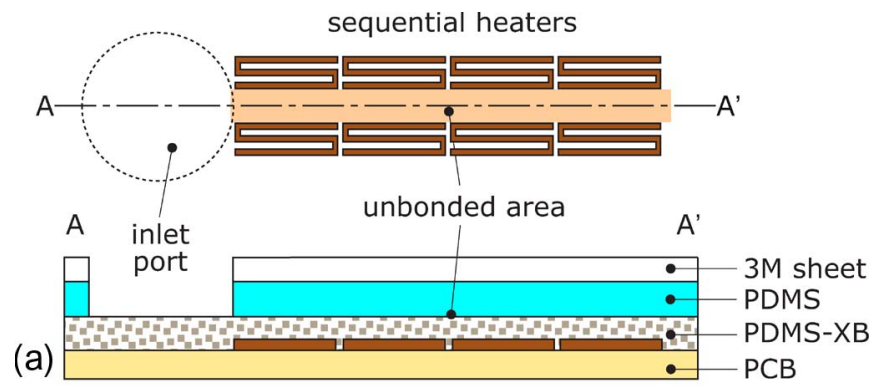

(b)

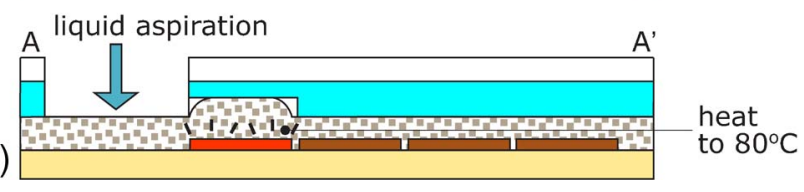

Fig. 11. (a) Schematic of an array of heaters which can be actuated sequentially to generate a fluidic pathway on demand. (b) Such an approach allows for increased thermal isolation by aspiration of an airplug first, or for bubble-free priming of the microchannel.

liquid. Furthermore, such a design can also be used for the bubble-free priming of a microchannel, due to the consecutively actuated heaters.

\section{CONCLUSION}

For the first time, we have shown active liquid aspiration units for effective liquid aspiration and release, which can be fabricated to operate in vertical as well as lateral direction. The devices are based on a single-use thermally expanding PDMS composite and feature a reinforced PDMS cover lid as well as selectively bonded PDMS areas to ensure device functionality. Two different approaches have successfully been applied to achieve such selective PDMS bonding on wafer-level scale, utilizing plasma-assisted PDMS bonding with an integrated antistiction layer and microcontact printing of PDMS curing agent.

Actively controlled vertical, as well as lateral, liquid aspiration and release has been demonstrated effectively to handle liquid volumes ranging from 28 to $815 \mathrm{~nL}$. The presented concept does not require external means for liquid actuation, it allows wafer-level fabrication and uses low-cost materials only. It potentially enables controlled and cost-effective transdermal microfluidic applications, point-of-care diagnostics, disposable biochips, or lab-on-a-chip applications.

\section{ACKNOWLEDGMENT}

The authors would like to thank Expancel, Sundsvall, Sweden, for kindly providing expandable microspheres.

\section{REFERENCES}

[1] D. J. Harrison and A. V. D. Berg, Eds., Micro Total Analysis Systems. Dordrecht, The Netherlands: Kluwer, 1998.

[2] A. Manz and H. Becker, Eds., Microsystem Technology in Chemistry and Life Sciences, Topics in Current Chemistry 194. Berlin, Germany: Springer-Verlag, 1998.

[3] P.-A. Auroux, D. Iossifidis, D. R. Reyes, and A. Manz, "Micro total analysis systems. 2. Analytical standard operations and applications," Anal. Chem., vol. 74, no. 12, pp. 2637-2652, Jun. 2002.

[4] A. van den Berg and T. Lammerink, Microsystem Technology in Chemistry and Life Sciences, Topics in Current Chemistry, vol. 194. Berlin,
Germany: Springer Verlag, 1998, ch. Micro Total Analysis Systems: Microfluidic Aspects, Integration Concept and Applications, pp. 21-49.

[5] S. Shoji and M. Esashi, "Microflow devices and systems," J. Micromech. Microeng., vol. 4, no. 4, pp. 157-171, Dec. 1994.

[6] H. Andersson, W. van der Wijngaart, P. Nilsson, P. Enoksson, and G. Stemme, "Valve-less diffuser micropump for microfluidic analytical systems," Sens. Actuators B, Chem., vol. 72, no. 3, pp. 259-265, Feb. 2001.

[7] M. A. Unger, H.-P. Chou, T. Thorsen, A. Scherer, and S. R. Quake, "Monolithic microfabricated valves and pumps by multilayer soft lithography," Science, vol. 288, no. 5463, pp. 113-116, Apr. 2000.

[8] R. F. Ismagilov, D. Rosmarin, P. J. A. Kenis, D. T. Chiu, W. Zhang, H. A. Stone, and G. M. Whitesides, "Pressure-driven laminar flow in tangential microchannels: An elastomeric microfluidic switch," Anal. Chem., vol. 73 , no. 19 , pp. 4682-4687, Oct. 2001.

[9] D. J. Beebe, J. S. Moore, J. M. Bauer, Q. Yu, R. H. Liu, C. Devadoss, and B.-H. Jo, "Functional hydrogel structures for autonomous flow control inside microfluidic channels," Nature, vol. 404, no. 6778, pp. 588-590, Apr. 2000.

[10] R. Liu, Q. Yu, and D. Beebe, "Fabrication and characterization of hydrogel-based microvalves," J. Microelectromech. Syst., vol. 11, no. 1, pp. 45-53, Feb. 2002.

[11] C. Yu, S. Mutlu, P. Selvaganapathy, C. Mastrangelo, F. Svec, and J. Frechet, "Flow control valves for analytical microfluidic chips without mechanical parts based on thermally responsive monolithic polymers," Anal. Chem., vol. 75, no. 8, pp. 1958-1961, Apr. 2003.

[12] D. Duffy, H. Gillis, J. Lin, N. Sheppard, and G. Kellogg, "Microfabricated centrifugal microfluidic systems: Characterization and multiple enzymatic assays," Anal. Chem., vol. 71, no. 20, pp. 4669-4678, 1999.

[13] S. Bohm, W. Olthuis, and P. Bergveld, "An integrated micromachined electrochemical pump and dosing system," Biomed. Microdevices, vol. 1, no. 2, pp. 121-130, Jun. 1999.

[14] J. Munyan, H. Fuentes, M. Draper, R. Kelly, and A. Woolley, "Electrically actuated, pressure-driven microfluidic pumps," Lab Chip, vol. 3, no. 4, pp. 217-220, 2003.

[15] Y. H. Choi, S. Son, and S. S. Lee, "Novel micropump using oxygen as pumping source," in Proc. MEMS, 2003, pp. 116-119.

[16] C.-C. Hong, S. Murugesan, S. Kim, G. Beaucage, J.-W. Choi, and C. Ahn, "A functional on-chip pressure generator using solid chemical propellant for disposable lab-on-a-chip," Lab Chip, vol. 3, no. 4, pp. 281-286, 2003

[17] E. Carlen and C. Mastrangelo, "Electrothermally activated paraffin microactuators," J. Microelectromech. Syst., vol. 11, no. 3, pp. 165-174, Jun. 2002.

[18] X. Yang, C. Grosjean, Y.-C. Tai, and C.-M. Ho, "A MEMS thermopneumatic silicone rubber membrane valve," Sens. Actuators A, Phys., vol. A64, no. 1, pp. 101-108, Jan. 1998.

[19] C. Cooney and B. Towe, "A thermopneumatic dispensing micropump," Sens. Actuators A, Phys., vol. 116, no. 3, pp. 519-524, Oct. 2004.

[20] P. Griss, H. Andersson, and G. Stemme, "Expandable microspheres for the handling of liquids," Lab Chip, vol. 2, no. 2, pp. 117-120, 2002

[21] N. Szita, R. A. Buser, and J. Dual, "Aspiration and dispensing of biological liquids in the micro- and submicroliter range with high precision," Biomed. Microdevices, vol. 3, no. 3, pp. 175-182, Sep. 2001.

[22] F. Foret, T. Rejtar, B. Zhang, and L. Karger, "Parallel sample loading and injection device for multichannel microfluidic devices," U.S. Patent 6939452 , Sep. 6, 2005.

[23] N. Ingenhoven, A. Hodac, and N. Schmid, "Device and system for dispensing and aspirating/dispensing liquid samples," European Patent 1333 926 B2, Sep. 22, 2004.

[24] B. Samel, P. Griss, and G. Stemme, "A thermally responsive PDMS composite and its microfluidic applications," J. Microelectromech. Syst., vol. 16, no. 1, pp. 50-57, Feb. 2007.

[25] B. Samel, J. Chretien, R. Yue, P. Griss, and G. Stemme, "Wafer-level process for single-use buckling-film microliter-range pumps," J. Microelectromech. Syst., vol. 16, no. 4, pp. 795-801, Aug. 2007.

[26] B. Samel, V. Nock, A. Russom, P. Griss, and G. Stemme, "A disposable lab-on-a-chip platform with embedded fluid actuators for active nanoliter liquid handling," Biomed. Microdevices, vol. 9, no. 1, pp. 61-67, Feb. 2007.

[27] B. Samel, M. K. Chowdhury, and G. Stemme, "The fabrication of microfluidic structures by means of full-wafer adhesive bonding using a poly(dimethylsiloxane) catalyst," J. Micromech. Microeng., vol. 17, no. 8, pp. 1710-1714, Aug. 2007.

[28] J. Lee, C. Park, and G. Whitesides, "Solvent compatibility of poly(dimethylsiloxane)-based microfluidic devices," Anal. Chem. vol. 75 , no. 23 , pp. 6544-6554, Dec. 2003. 


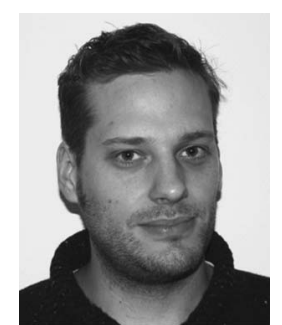

Björn Samel received the Dipl.-Ing.(FH) degree (with honors) in mechatronics from the University of Applied Sciences, Ulm, Germany, in 2002, and the $\mathrm{Ph} . \mathrm{D}$. degree in electrical engineering from the Microsystem Technology Laboratory, The Royal Institute of Technology, Stockholm, Sweden, in 2007.

Currently, he is working on the development of MEMS applications toward the fields of aerospace and outer space with Ångström Aerospace Corporation, Uppsala, Sweden.

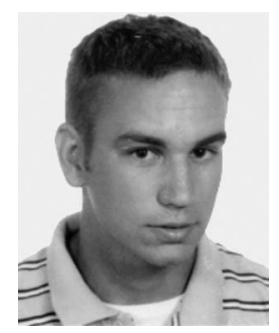

Niklas Sandström received the M.Sc. degree in engineering biology from Linköping University, Linköping, Sweden, in 2007. He is currently working toward the Ph.D. degree in the School of Electrical Engineering, The Royal Institute of Technology, Stockholm, Sweden.

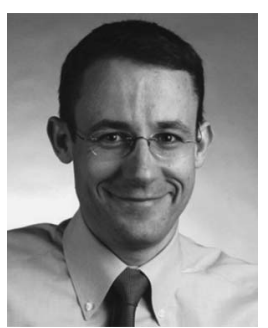

Patrick Griss received the M.Sc. degree in microengineering from the Swiss Federal Institute of Technology, Lausanne, Switzerland, in 1999, and the $\mathrm{Ph} . \mathrm{D}$. degree in electrical engineering from The Royal Institute of Technology, Stockholm, Sweden, in 2002.

After four years of research in the in vitro diagnostic field at Roche Diagnostics, he is currently heading the Technology and Integration Business Unit at Zühlke Engineering AG, a technology consulting company based in Zürich, Switzerland.

Dr. Griss, together with two colleagues, won the Swedish Innovation Cup in 2001.

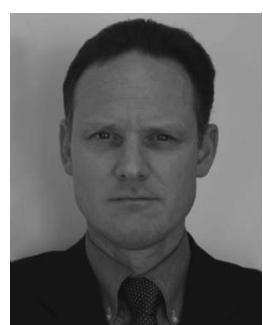

Göran Stemme (M'98-SM'04-F'06) received the M.Sc. degree in electrical engineering and the Ph.D degree in solid state electronics from Chalmers University of Technology, Gothenburg, Sweden, in 1981 and 1987, respectively.

In 1981, he was with the Department of Solid State Electronics, Chalmers University of Technology. There, in 1990, he became an Associate Professor (Docent) heading the silicon sensor research group. Since 1991, he has been Professor at The Royal Institute of Technology, Stockholm, Sweden, where he currently heads the Microsystem Technology Laboratory in the School of Electrical Engineering. He has published more than 200 research journal and conference papers and has more than 12 patent proposals or granted patents.

Dr. Stemme was a member of the International Steering Committee of the conference series IEEE Microelectromechanical Systems between 1995 and 2001, and he was General Cochair of that conference in 1998. $\mathrm{He}$ has been a member of the Editorial Board of the JOURNAL OF MicroelectromechaniCAL SyStEMS since 1997 and was a member of the Editorial Board of the Royal Society of Chemistry journal Lab On A Chip between 2000 and 2005. Since 2005, he has been a member of the International Steering Committee of the conference series IEEE Solid-State Sensors, Actuators and Microsystems (Transducers). He is a member of the Royal Swedish Academy of Sciences. 